\title{
TRAJECTORY PLANNING OF MOBILE ROBOT MOVEMENT IN UNKNOWN ENVIRONMENT
}

\author{
Andrius Nemeiksis, Vitalijs Osadcuks \\ Latvia University of Agriculture \\ nemeiksis.andrius@1lu.lv, vitalijs.osadcuks@1lu.lv
}

\begin{abstract}
The application of mobile robots is becoming more and more common in order to perform various tasks under conditions where the presence of the human in their site is impossible on safety grounds or unacceptable due to the reduction of the productivity of the technological equipment served by them. The objective of robot movement planning is to guarantee the desirable robot movement path as it moves across the planned path on the basis of controlling impacts, i.e. sensors. Numerous investigations were carried out under unknown environment conditions, which were intended for addressing the problem of robot movement without colliding with obstacles in its path by employing various navigation methods. The purpose of this paper is to analyse navigation methods employed for addressing the problem of robot movement without colliding with obstacles in its path under unknown environment conditions. The paper analyses the multi-agent system framework, generalized agent control system framework, local path planning algorithm for robots in an unknown two-dimensional space and other related questions in order to reach the above-mentioned purpose.
\end{abstract}

Keywords: geometric path planning method, multi-agent system, artificial potential method, global planning, local planning.

\section{Introduction}

The development of mobile robotics is determined by the desire to replace men at hard and routine, dangerous and responsible work, i.e. in warehouses for efficiently moving materials from stocking shelves to order fulfilment zones, in hospitals for moving materials, in military for clearing mines, in household for vacuuming or gardening etc. The most important characteristics of such robot are mobility and autonomy. In this case, autonomy is necessary in order to guarantee the movement in an unknown environment so that the mobile robot (MR) is able to independently move in an unknown environment performing the task set.

The mobile robot movement planning is the most important issue of the autonomous robototechnical system control and one of the fields of modern science and practice most actively investigated. The solution to the task of robot movement planning colliding encompasses questions related with scientific fields such as artificial intelligence, computational geometry, computer simulation and the theory of automatic control. The automation of the movement planning process by also minimising the time costs for preparation and completion operations as well as by accelerating the process of switching the robot from one production task to another constitute the basis for flexible production organisation.

Various achievements of scientists in this field enabled to determine the priority trends of mobile robot development. The main design tasks of mobile robot movement in unknown environment conditions are provided in Figure $1[1 ; 2]$.

It is easy to understand that the solution to the tasks listed above (Figure 1) requires the solution to a variety of main autonomous mobile robot tasks. First, the robot shall plan a fast and passable route in order to move in an unknown environment. For this purpose it shall be able to precisely follow its location and find a way as well as to identify all obstacles in the way. However, even a well-defined route is worthless, if the robot is not able to identify its present location and the further direction. Furthermore, the mobile robot shall manoeuvre in order to go round the obstacles, especially when moving fast and when the road is slippery. Therefore, the algorithmic equipment of their control systems shall include the following characteristics [3]:

1. To generate the internal description ("map") of the current state of the space, in which the robot movement is taking place, by specifying the movement target and the position of obstacles interfering with the required movements;

2. To correct the movement path on the basis of prior and posterior data stored in the memory as well as the existing sensor data;

3. To move on the basis of the planned path [4]. 
In this case, the analysis of the development of mobile robots that move in an unknown environment shows that the main problem of mobile robot moving independently, without human intervention, is the navigation task solution, which refers to the determination of the mobile robot position in the site, i.e. its localisation and the environmental concept, preparation of the description, i.e. localization. Information on the current robot position is necessary in solving the majority of control tasks that arise. In the case of successful navigation in space, the robot control system shall be able to calculate the route, to control the movement parameters (to provide the wheel turn angle and the speed of their rotation), to correctly interpret the environmental data received from the sensors and to constantly follow its coordinates.

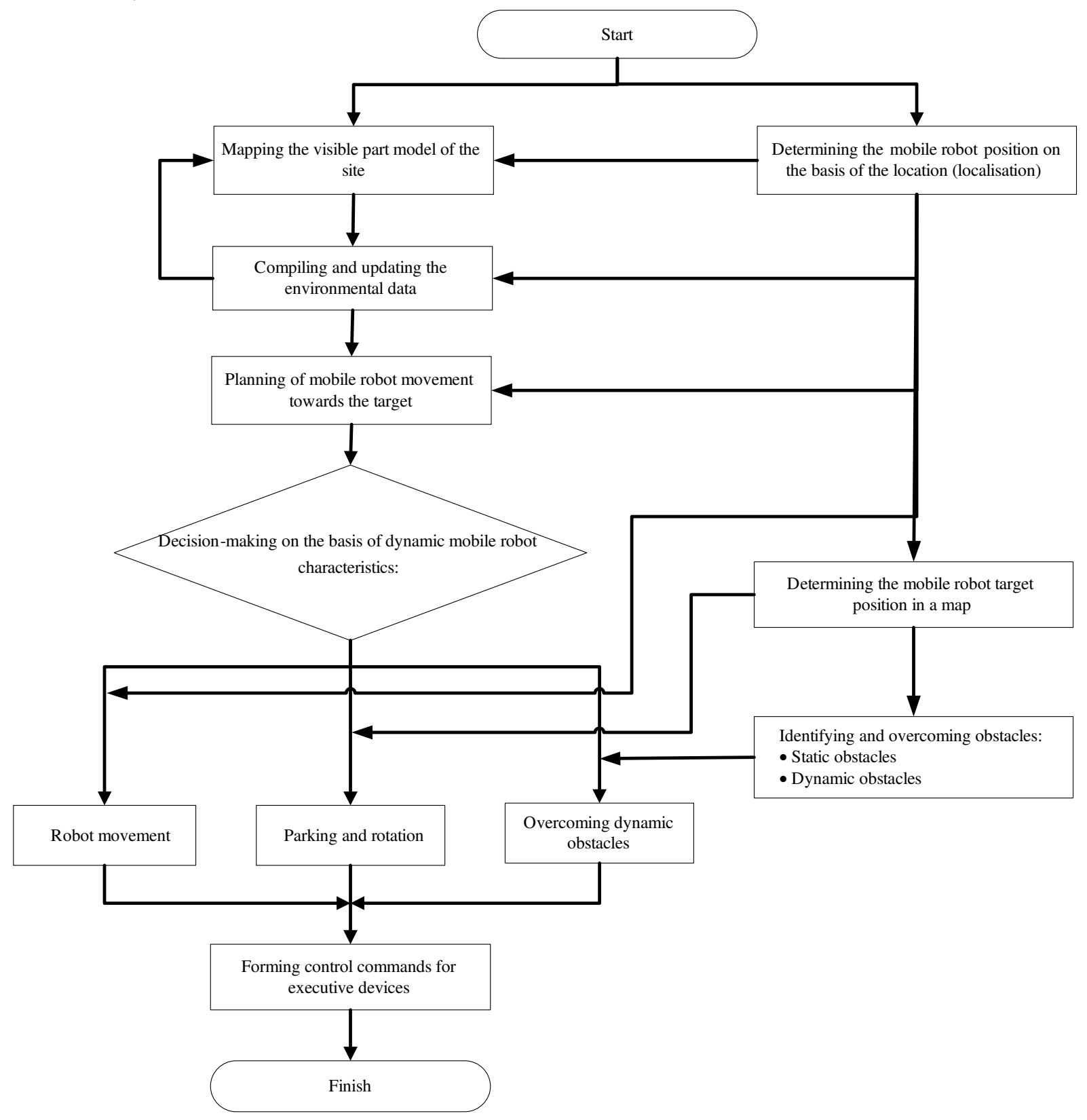

\section{Fig. 1. Main design tasks of mobile robot movement in an unknown environment} (made by the authors)

Regardless of the construction differences of autonomous mobile robots, their developers encounter general interrelated tasks: navigation of mobile robots moving in an unknown environment and the movement control. The autonomous mobile robot navigation and movement control problem is constituted by two tasks as seen from Figure 1. The first task is to visualise the environment and to be able to find the way in it: to generate location maps and to determine the MR position in the site. It is referred to as the localization problem in scientific literature [5]. Another task is to guarantee the 
mobile robot movement in an unknown environment: to create a robot movement path, to overcome the paths set, to correct the movement path on the basis of sensor data and to safely go round the obstacles. It is referred to as the exploration problem in scientific literature [6]. The integrated mobile robot navigation and movement control system shall be able to model the environment, to set the robot movement parameters and to control its movement.

Therefore, depending on the robot sensors, the formulation of these tasks requires the development of robot navigation and movement control methods, because there are vast different areas of usage of mobile robots in practice, i.e. in warehouses for efficiently moving materials from stocking shelves to order fulfilment zones, in hospitals for moving materials, in military for clearing mines, in household for vacuuming or gardening etc.

The analysis of the results of these studies shows that the problem of the mobile robot motion in an unknown environment is solved by estimating the robot movement direction in the operative local planning mode and the speed of the process determines the possibility to solve the problem of the mobile robot motion in an environment with mobile obstacles. The purpose of this paper is to analyse navigation methods employed for addressing the problem of robot movement without colliding with obstacles in its path under unknown environment conditions. We analyse the multi-agent system framework, generalised agent control system framework, local path planning algorithm for robots in an unknown two-dimensional space and other related questions in order to reach the above-mentioned purpose.

\section{Materials and methods}

The main topics of the mobile robot path planning are presented in various sources. Today, there are various methods for trajectory planning, but in the present cases it is significant to pay attention to their distinction between global and local methods groups, which contain some respective advantages and disadvantages. First of all, the research of the global path planning methods covers different planning techniques by using autonomous mode in a known static environment. Known static environment is interpreted as the robot surrounding environment, where the obstacles in the operational area are familiar in their form, size, and location [7]. The advantage of the Global path planning methods is that future robot trajectory is calculated in autonomous mode, but in this case it is impossible to evaluate all possible system components, as well as together with the increasing complexity of the system, there is a rapid increasing of calculation complexity, which does not meet the requirements of bandwidth that is necessary to the real-time mode control in unfamiliar environments. However, the local trajectory planning methods shall be evaluated as being the ambiguously one, i. e. since they are much simpler mathematical apparatus, requiring less time consumption calculations in comparison with the global trajectory planning methods, but, on the other hand, the optimal solution as result of such trajectory planning methods cannot exist because of the relatively limited information about the environment, which is used to set the robot movement path. As consequences of that, the local trajectory planning methods usage in mobile robot control is often ineffective when it comes to choose the trajectory between several obstacles arranged close to each other.

The first and most widespread method for the solution to the mobile robot path planning problem in an unknown environment is the geometric path planning method. In order to avoid the robot collision with the nearest obstacle, we need a continuous obstacle movement control. It should be noted that the geometric method is easy to use, but it is not always suitable, for instance, if the robot operational area has several closely located obstacles. However, here closely positioned obstacles can be easily distinguished by more precise sensors, on the one hand, if the high costs are not the issue, while on the other hand - a good control algorithm can help too, because the costs consist of the implementation costs $[7 ; 8]$

Another method for the solution to the mobile robot path planning problem in an unknown environment is the multi-agent system, used to identify all the possible environment settings and estimate the corresponding robot actions. The multi-agent system is a new and important modern research technology in the field of artificial intelligence. An agent has the following characteristics:

- autonomy - ability to independently and automatically track the environment; 
- communication - ability to communicate with other agents or users (operators);

- reactivity - ability to assess the information regarding the environment and react to it;

- flexibility - ability to perform various tasks.

So, the agent-based system can operate in an unknown dynamic environment. In this case, the mobile robot motion without running into obstacles is the result of the path formation, when an adequate action is performed in every possible situation. The multi-agent system is suitable to plan the mobile robot motion in the indoor environment [7, 9].

Figure 2 presents the general multi-agent system framework for the robot motion planning in an unknown environment.

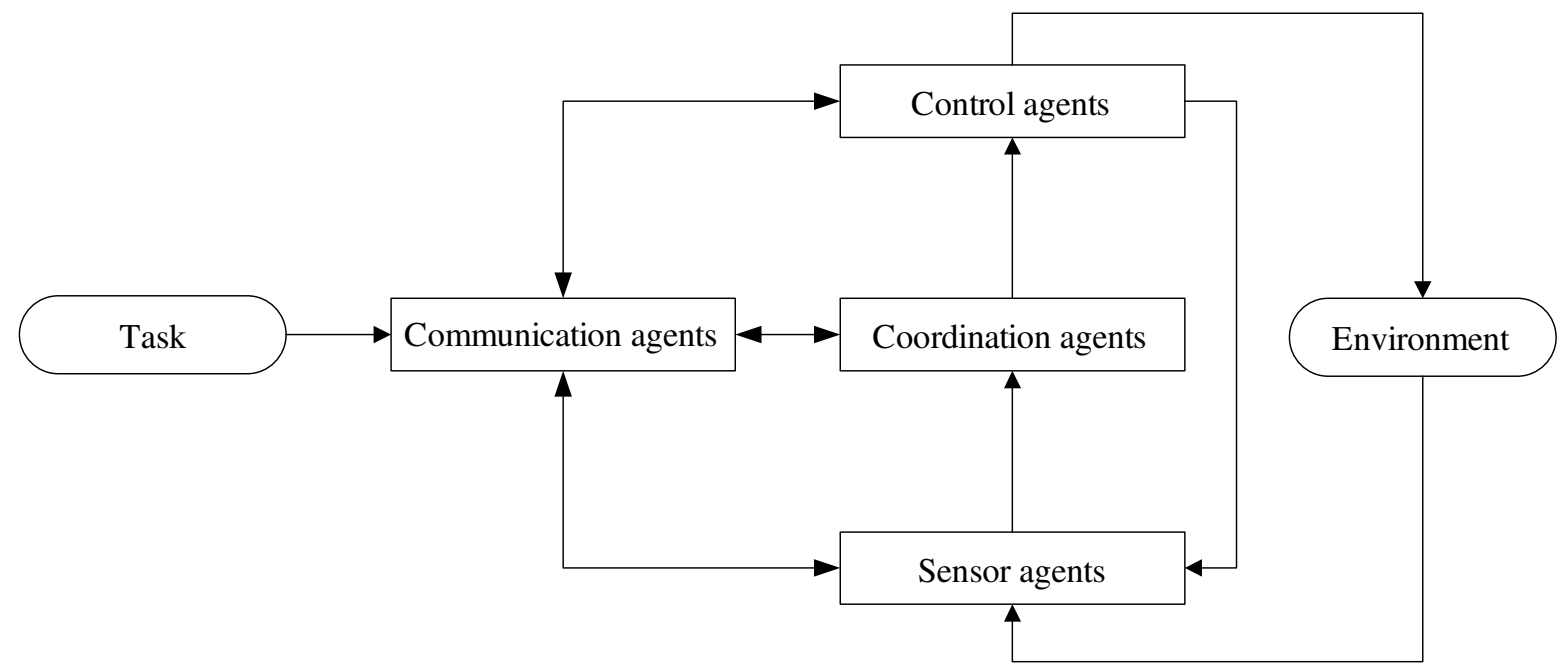

Fig. 2. General multi-agent system framework [7]

Figure 3 presents several agents that are widely used in the mobile robot motion planning and control system.
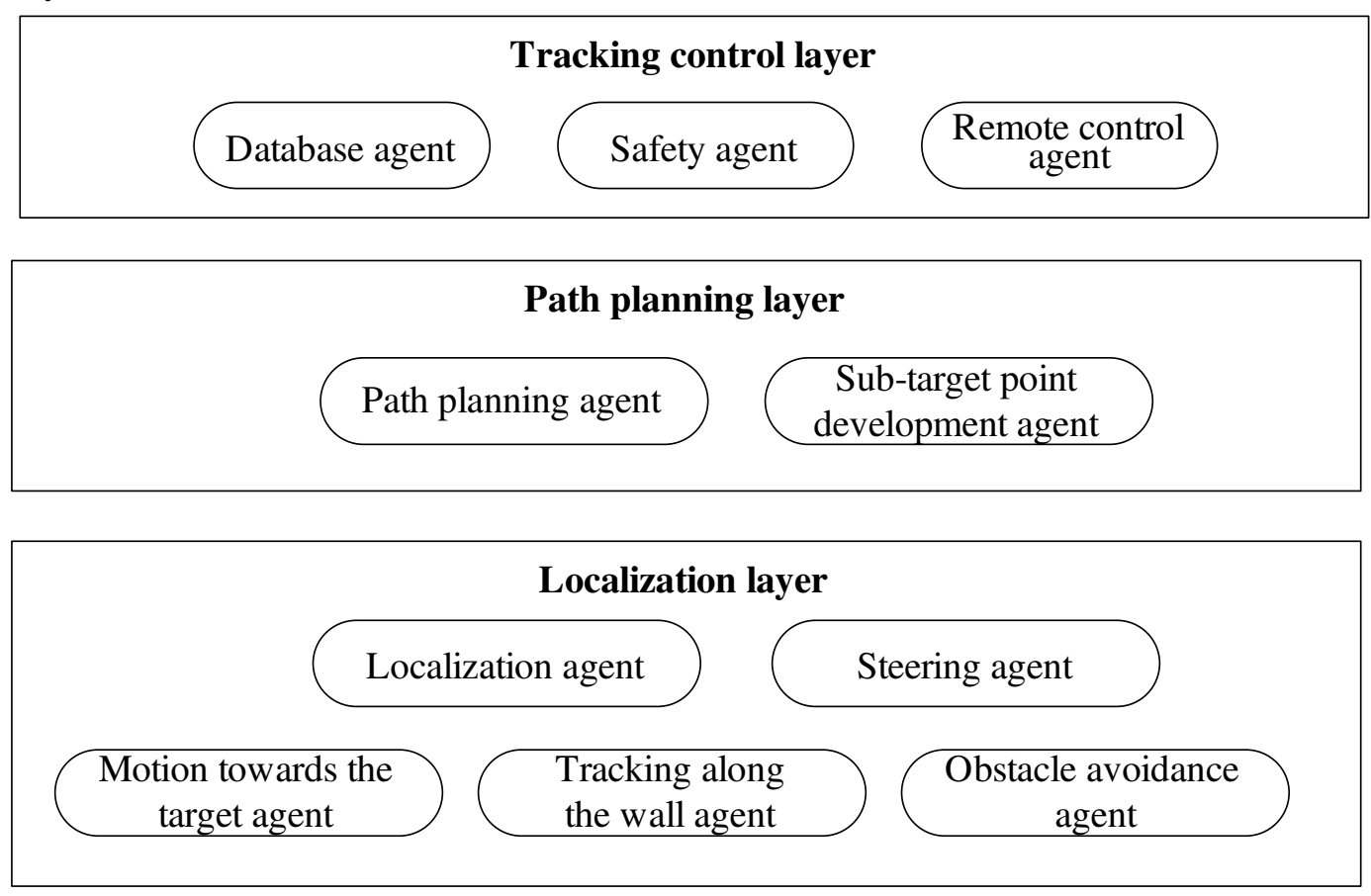

Fig. 3. Agent types [9]

Talking about the use of such agents, the task to plan the path towards the target with suddenly appearing obstacles may also arise to the agents operating in underwater conditions. For instance, we 
will analyse the unmanned bathyscaphe type agent. Such agents are widely applied while exploring the bottom of the sea, searching for underwater objects, maintaining underwater communication devices, etc. The path towards the target for such agents can be set by the operator, working with the control mode, or it can be set automatically in accordance with the data provided by the depth map.

However, while moving along the planned path towards the target object, the agent may face an unexpected obstacle, such as an underwater relief fragment, which is not on the map, or the sunken ship, as depicted in Figure 4.

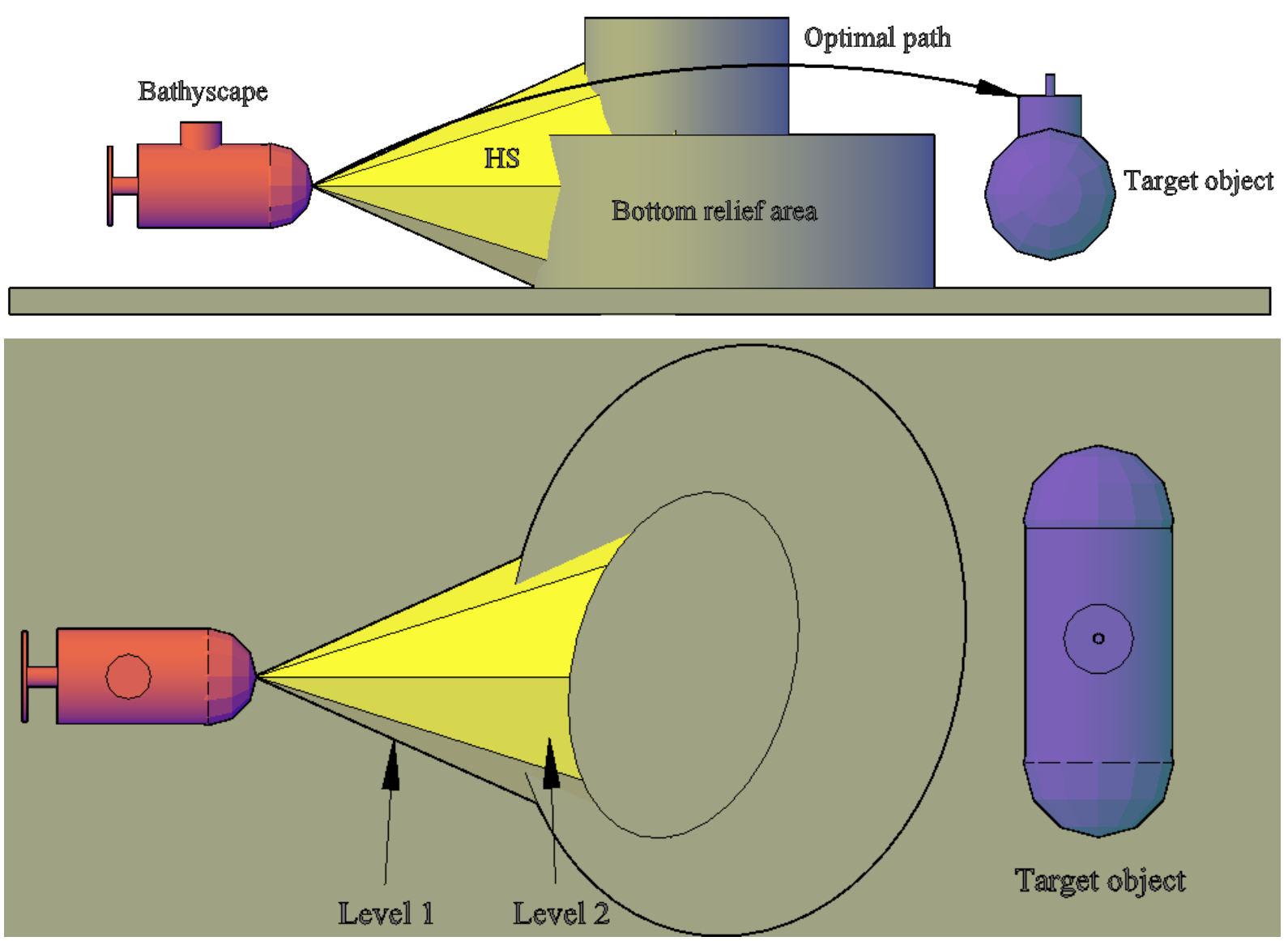

Fig. 4. Localization task example for an underwater agent (made by the authors)

In such situations the task may arise to form the outdoor environment accessibility model along the agent motion direction in accordance with the information provided by the hydro-acoustic system (HS), as well as to plan and estimate an optimal path and its formation rate. Moreover, all the discussed actions must be planned and improved in real-time, which is a difficult task even for a bathyscaphe operating in manual control mode. This problem can be solved by integrating the agent control block into the system and that automatically solves localization tasks using also specific mathematical or AI methods.

An agent, operating in above-water environment, may face underwater obstacles of artificial origin, for instance, lying at the bottom in various distances from the agent and causing different degrees of hazard.

So, we can distinguish the following localization tasks performed by an agent in various environments:

1. Accessibility (free for movement places) localization of an outdoor environment, which is not formalised beforehand;

2. Modelling of accessibility of an outdoor environment towards the agent moving direction;

3. Determining its own position in the map based on the information from the environment;

4. Planning the path towards the target under the localization hazard (obstacles) conditions. 
It should be noted that the quality of determining its own position highly influences other localization task solutions. It is presumed that if an agent operates under the conditions of an outdoor environment, which is not formalised beforehand, it is unable to estimate its own position coordinates in that environment, based on the data from the satellite position estimation systems such GPS (in general GNS - global navigation system) or GLONASS. That is why the foregoing task is usually solved based on the data regarding the agent motion obtained by the odometric subsystem and momentum localization methods. However, in this case, major errors may occur, which increase as the time passes, and the rate of which is hard to estimate. The mentioned circumstances encourage the creation of new algorithms in order to solve this task, which aim is to increase the quality of the $\mathrm{OE}$ control system operation.

Generalised agent control system framework, performing the mentioned tasks, can be visualised as presented in Figure 5 [10].

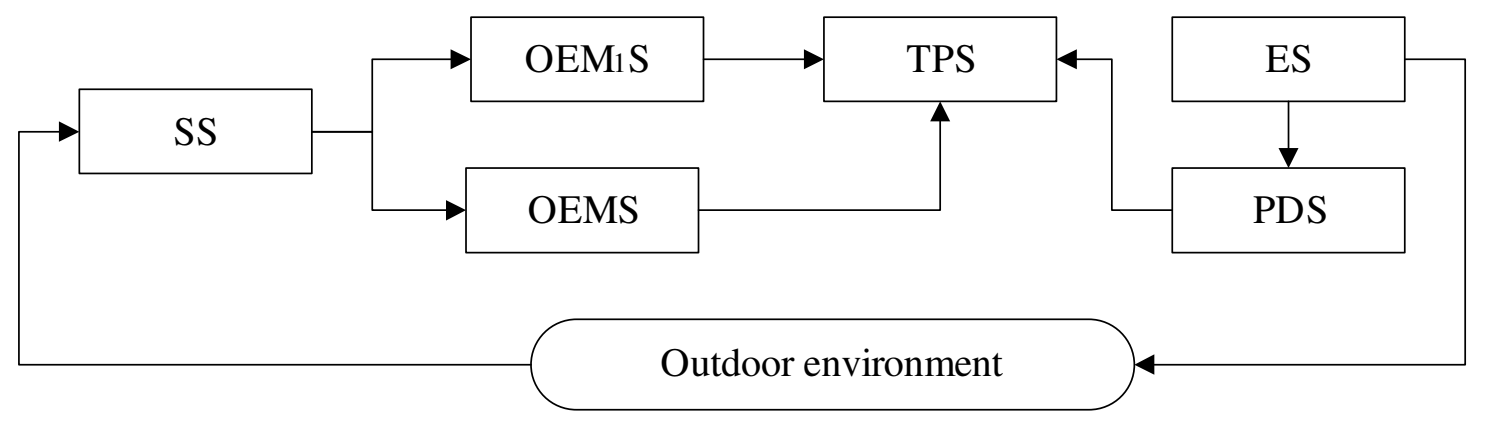

Fig. 5. Generalised agent control system framework (made by the authors)

Markings used in the figure above: SS - sensor subsystem; OEMS - outdoor environment localization subsystem; $\mathrm{OEM}_{1} \mathrm{~S}$ - outdoor environment modelling subsystem; TPS - toward-the-target path planning subsystem; PDS - position determination subsystem; ES - executive subsystem.

Yet, another method in order to solve the problem of the mobile robot motion planning in an unknown environment is the artificial potential method, which initially was intended to use within the local method limits, when the robot path planning without facing the obstacles was implemented by an operator. To optimize the process it is appropriate to apply the fuzzy logic model $[11 ; 12]$ or the Spiking Neuron Algorithm with Axonal Delays method [13].

The high scale of optimization of planning the mobile robot motion is only possible in autonomous mode under the known environment conditions, when the robot moves along the planned path, while in the unknown environment the scale of optimization decreases, because at least a small portion of the unknown environment is known after an obstacle is encountered in the robot moving. Currently, two methods are applied in order to optimize the planning the mobile robot motion, if the robot operational area has only immobile obstacles (in static environment):

1. The first method is applied when there is multiple number of robot path points, while the second method is applied when we have the initial and final path points. In this case, non-linear optimization of planning the mobile robot motion estimates the respective kinematic and dynamic limitations. Within the limits of the first method, the optimization of planning the mobile robot motion involves the motion vector formation during every time interval.

2. The second method offers the implementation of this procedure based on interpolation coefficients $[14 ; 15]$.

It should be noted that the optimisation of the mobile robot path planning is impossible under the unknown environment conditions, as its performance requires the information regarding obstacles in the robot operational area. Also, the planning optimisation is not applied in the cases when a mobile robot moves in a dynamic environment, as the respective calculations and their results are used for immobile obstacles only.

So, all the three previously discussed trajectory planning methods are the real measures to deal with issues of mobile robot motion planning in an unfamiliar environment, but they have particular specifics, what must be taken into account in decision-making on this question. 


\section{Results and discussion}

Autonomous robotic systems, performing the planned tasks in an unknown environment, have a high demand in the modern industry and other fields of economics (e.g., in warehouses for efficiently moving materials from stocking shelves to order fulfilment zones, in hospitals for moving materials, in military for clearing mines, in household for vacuuming or gardening etc.), which in turn shows the relevance of the research concerning the problem of the mobile robot motion planning without running into unknown obstacles [16;17].

Let us analyse the global planning task, within the limits of which the mobile robot path is treated as the sequence of its path segments or transit points, in more detail. It should be noted that the simple path configurations, for instance, straight lines, are formed by connecting these points. However, the changes in the environment can make it impossible to calculate all the situations the mobile robot may face. In that case, the robot will not be able to finish the planned path. The solution to this problem is to apply the local planning, where the priority is given to the obstacle avoidance, and reaching the transit or target point becomes a secondary task [12; 14]. So, the mobile robot motion planning in an unknown environment is very much different from the motion planning in a known environment, since unknown environment turns to known in small scale, when the obstacle is identified and replanning is necessary. The main task of the first method is to implement the planning process in accordance with the local information about the environment obtained from the sensors, which step by step familiarize with the robot operational area $[18 ; 19]$.

Nowadays, the local planning is considered to be a relevant issue in robotics. In addition, the method application for the control in the real-time mode with time limitations is widely spreading. The methods of the local mobile robot motion planning require relatively small time scales, and that helps the robot operate faster and allows it to perform various tasks in critical situations in an unknown environment, for instance, in warfare or in the vehicle use [20]. The local planning methods differ from the global planning methods in the way they allow to control the mobile robot of any configuration. It should be noted that the main drawback of the local planning methods is the possibility that the task will be impossible to perform, if the robot faces several closely located obstacles.

While preparing the task of the mobile robot motion control in an unknown dynamic environment, the local planning methods are an additional part of the multi-stage planning systems. In every stage of these systems the functional module performs such functions as the local path planning, obstacle collision avoidance assurance, and actuator control [7].

By applying the local motion planning, the avoidance of the robot collision with obstacles is analysed as the control problem. The local planning methods do not mean to replace the global safe path planning. Their use is based on the control possibilities in the real-time mode, when the sensor information about the environment becomes the basis for the robot motion adjustment due to the changes in its operational area [21].

The solution to the problem of the robot motion planning in an unknown environment encompasses the planning of its movements from the initial point to the target point without running into obstacles. In this case, the robot is not only equipped with the sensors that measure the distance to the objects, but it can also determine its own position and orientation. We can analyse the following local path planning algorithm for robots in an unknown environment as an example (Figure 5).

The mobile robot moves along the path $\mathrm{PiP}_{\mathrm{i}+1}$, connecting the initial and target points. If there are no obstacles on its way, the algorithm is considered completed the moment the robot reaches the target point. On the other hand, if the distance sensors detect an obstacle on the way $P_{i}$ to $P_{i+1}$, the mobile robot avoids the obstacle collision and reaches the point $P_{i+1}$ using the shortest path [22].

O. Khatib researched the method of the local mobile robot motion planning in an unknown environment in the real-time mode, which eliminates the robot collision with obstacles. The principle of this method is the use of the artificial potential field that changes over time. In case the safe path is found, the artificial potential field uses the local information. The target point generates the attracting potential, while the obstacle generates the repelling potential. The negative outcome gradient of the potential field determines the most probable robot motion direction. The main advantage of the Khatib 
method is that its application allows the robot to expeditiously react to unexpected situations in the environment [16].

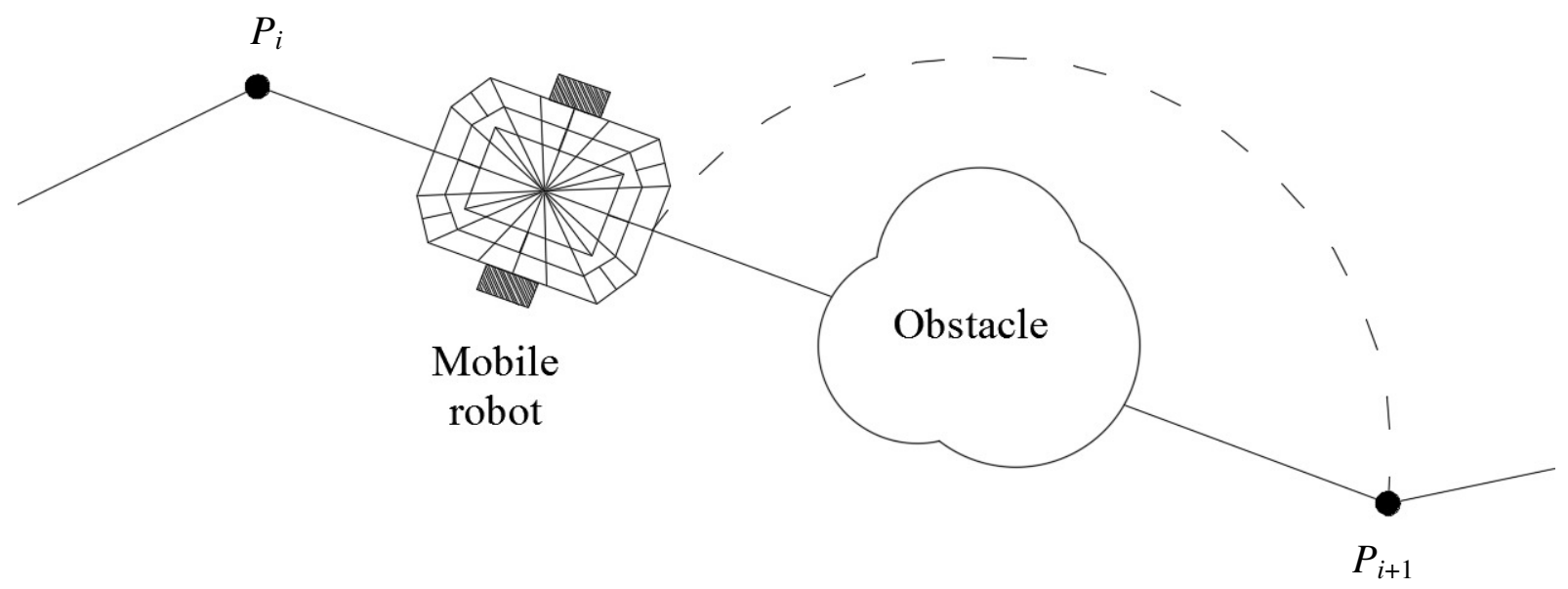

Fig. 6. Local path planning algorithm for robots in an unknown two-dimensional space (made by the authors)

However, Fir A. Rahim thought that just by applying the artificial potential method there is usually no guarantee that the obstacle-free path will be found [7]. Correspondingly, Y. Fu, B. Jin and S. Wang point out that the artificial potential method is impossible to use in an environment with mobile obstacles, as this method requires specific information about them [23].

R. A. Brooks applied the force-field concept for a mobile robot with ultrasonic sensors to avoid obstacles. The information obtained by the sensors was used to calculate the resultant repelling force [9]. J. Borenstein and Y. Koren suggested the vector field histogram method for a fast moving robot with ultrasonic sensors [24]. However, these methods have drawbacks. For instance, it is hard to estimate the force coefficients, which influence the mobile robot velocity and direction in dynamic environment.

Mark Leyden introduced the method of the mobile robot motion planning in an unknown environment, which includes two types of behaviour: avoiding the obstacles and reaching the target. In order to combine some of the robot's behavioural choices more easily, during its movement from the initial point to the target point, fuzzy logic was applied. The main advantage of the Mark Leyden method is that the robot motion is safe and even [25]. However, this method is inaccurate in more complex environments.

Along with the Mark Leyden concept, some scientists developed the methods of the robot motion planning in an unknown environment by using artificial intelligence and many behavioural choices. HartmutSurmann and JorgHuser suggested the mobile robot motion planning system based on fuzzy logic agents of the multi-agent system. The complex planning is achieved by combining local actions and global algorithms in a fuzzy agent. Various types of behaviour are identified by using a fuzzy agent base and storing in the state variables. These state variables activate various fuzzy agent bases that change the behaviour of a fuzzy agent [26]. The location of usage of fuzzy blocks of each agent and the task solution of robot motion planning in each particular situation allow to increase the accuracy and efficiency of robot motion planning [39].

In general, it could be stated that the problem of the robot motion planning in a known environment is usually solved by using the autonomous global path planning methods. The investigated methods, including the path map method, the division into elements method, and the artificial potential method, require relatively large time scales for the calculations. That is why they cannot be applied for the operative path planning or under the environment conditions with mobile obstacles.

\section{Conclusions}

To summarise the analysis of the robot motion planning in an unknown environment, discussed in this chapter, the following conclusions can be drawn. 
1. All the real-time mode motion planning models without any exception use sensor systems, necessary to estimate the local environment of a robot. These models are based on the most popular and successful geometric methods as the configuration space, the artificial potential method and the multi-agent system. The paper analysed the interactions of these various robot control methods and different unfamiliar environments.

2. The environmental model update, by scanning the local information from the distance sensors, is performed step by step or during every program iteration.

3. The relevant information, received from the distance sensors, is used in the decision making stage, when the obstacle-free path points in the robot operational area are identified. Due to the analysed planning stage projection, the use of the intelligent principles of its preparation is a must.

4. So, the problem of the mobile robot motion in an unknown environment is solved by estimating the robot movement direction in the operative local planning mode. Correspondingly, the speed of the process determines the possibility to solve the problem of the mobile robot motion in an environment with mobile obstacles.

\section{References}

1. Hartanto R. A hybrid deliberative layer for robotic agents: fusing DL reasoning with HTN planning in autonomous robots. Springer-Verlag. Berlin: Heidelberg, 2011. 215 p.

2. Samaka M. Robot task-level programming language and simulation. International journal of computer, electrical, automation, control and information engineering, vol. 1, no. 9, 2007, pp. 2843-2847.

3. González R, Rodriguez, F., Guzmán, J.L. Autonomous tracked robots in planar off-road conditions. Modelling, Localization, and Motion Control. Springer-Verlag. Berlin: Heidelberg, 2014. $119 \mathrm{p}$.

4. Nikitenko A., Liekna A., Andersone I., Ekmanis M., Urtans E. Mobile robot path planning for indoor use. Proceedings of $14 \mathrm{~h}$ International scientific conference „Engineering for rural development“, May 29-30, 2014, Jelgava, Latvia, pp. 366-372.

5. González R., Fiacchini M., Guzmán J.L., Alamo T., Rodrıguez F. Robust tube-based predictive control for mobile robots in off-road conditions. Robotics and autonomous systems, vol. 59, no. 10, 2011, pp. 711-726.

6. Siciliano B. Handbook of Robotics. Springer-Verlag. Berlin: Heidelberg, 2008. 1611 p.

7. Jeffril M.A., Sariff N. The integration of fuzzy logic and artificial neural network methods for mobile robot obstacle avoidance in a static environment. Proceedings of 2013 IEEE 3rd International Conference on System Engineering and Technology, August 19-20, 2013, Shah Alam, Malaysia, pp. 325-330

8. Mohanty P.K., Parhi D.R., Jha A.K., Pandey A. Path planning of an autonomous mobile robot using adaptive network based fuzzy controller. Proceedings of $2013 \mathrm{3rd}$ IEEE International „Advance Computing Conference (IACC)”, February 22-23, 2013, Ghaziabad, India, pp. 651-656

9. Gao Q., Cho Y.I. A Dynamic ontology-based multi-agent context-awareness user profile construction method for personalized information retrieval. International journal of Fuzzy logic and intelligent systems, vol. 12, no. 4, 2012, pp. 270-276.

10. Siegwart R., Nourbakhsh I., Scaramuzza D. Introduction to autonomous mobile robots. The MIT Press 2011. 488 p.

11. Boonphoapichart, S., Komada, S., Hod, T. Robot's motion decision-making system in unknown environment and its application to a mobile robot. Proceedings of 2002 IEEE International Conference "Industrial Technology“, December 11-14, 2002, Tsu, Japan, pp. 18-23.

12. Zhang H., Shi Z., Wei X. A rapid path planning adaptive optimization algorithm based on fuzzyneural network for multi-robot systems. Proceedings of "International conference on cyberspace technology (CCT 2013)", November 22-23, 2013, Beijing, China, pp. 32-38.

13. Ponulak F., Hopfield J.J. Rapid, parallel path planning by propagating wavefronts of spiking neural activity. Frontiers in computational neuroscience, vol. 7, no. 98, 2013, USA, pp. 1-14.

14. Han X., Payandeh S. Experimental design and analysis in kinematic-based localization in wireless mobile platform network. Proceedings of 2012 IEEE International Systems Conference "SysCon 2012”, March 19-22, 2012, Vancouver, Canada, pp. 1-6. 
15. Li Z., Deng J., Lu R., Xu Y., Bai J., Su C.Y. Trajectory-tracking control of mobile robot systems incorporating neural-dynamic optimized model predictive approach. IEEE Transactions on Systems, Man, and Cybernetics: Systems, vol. 46, no. 6, 2016, pp. 740-749.

16. Nagata F., Otsuka A., Watanabe K. Network-based subsumption architecture for multiple mobile robots system. Proceedings of the 6th International Conference on "Soft Computing and Intelligent Systems" and the 13th International Symposium on "Advanced Intelligent Systems" (SCIS-ISIS 2012), November 20-24, 2012, Kobe, Japan, pp. 187-192.

17. Yu Y., Gupta K. C-space entropy: A measure for view planning and exploration for general robotsensor systems in unknown environments. The International Journal of Robotics Research, vol. 23, no. 12, 2004, pp. 1197-1223.

18. Tingbin C., Qisong Z. Robot motion planning based on improved artificial potential field. Proceedings of 2013 3rd International Conference on "Computer Science and Network Technology", October 12-13, Dalian, China, 2013, pp. 1208-1211.

19. Nagata F., Otsuka A., Watanabe K., Habib M.K. Multiple mobile robots system with networkbased subsumption architecture. International Journal of Mechatronics and Manufacturing Systems, vol. 6, no. 1, 2013, pp. 57-71.

20. Li T., Yuan G., Duan Q. Navigation technology of autonomous mobile robots in unknown environments. Proceedings of 2010 IEEE/ASME International Conference on Mechatronic and Embedded Systems and Applications, July 15-17, 2010, Qingdao, ShanDong, China, pp. 533-538.

21. Afaghani A.Y., Aiyama Y. On-line collision avoidance between two robot manipulators using collision map and simple Escaping method. Proceedings of the 2013 IEEE/SICE International Symposium on System Integration, December 15-17, 2013, Kobe, Japan, pp. 105-110.

22. Tzafestas S.G. Introduction to mobile robot control. Elsevier, Athens, Greece, 2013. 750 p.

23. Roy S., Banerjee D., Majumder C.G., Konar A., Janarthanan R. Sensory-data extraction based real time mobile robot motion planning using fuzzy logic. Proceedings of the 2012 12th International Conference on Hybrid Intelligent Systems (HIS), December 4-7, 2012, Pune, India, pp. 468-473.

24. Ferrier J.L., Bernard A., Gusikhin O., Madani K. Informatics in Control, Automation and Robotics. Proceedings of the 8th International Conference on Informatics in Control Automation and Robotics, July 28-31, 2012, Rome, Italy, pp. 651-656.

25. Pandey A., Sonkar R.K., Pandey K.K., Parhi D.R.. Path planning navigation of mobile robot with obstacles avoidance using fuzzy logic controller. Proceedings of the 2014 IEEE 8th International Conference on Intelligent Systems and Control (ISCO), January 10-11, 2014, Coimbatore, India, pp. 39-41.

26. Johnson J., Godwin D.J. Indoor navigation of mobile robot using fuzzy logic controller. Proceedings of the 2015 3rd International Conference on Signal Processing, Communication and Networking (ICSCN), March 26-28, 2015, Chennai, India, pp. 1-7. 